\title{
Bearing Diagnostics of Hydro Power Plants Using Wavelet Packet Transform and a Hidden Markov Model with Orbit Curves
}

\author{
Gabriel Pino $\mathbb{D}^{1},{ }^{1}$ José Roberto Ribas $\mathbb{D}^{2},{ }^{2}$ and Luciana Fernandes Guimarães $\mathbb{D}^{2}$ \\ ${ }^{1}$ Universidade de São Paulo, São Paulo, SP, Brazil \\ ${ }^{2}$ Universidade Federal do Rio de Janeiro, Rio de Janeiro, RJ, Brazil \\ Correspondence should be addressed to Gabriel Pino; gabriel.pino@usp.br
}

Received 15 August 2017; Revised 23 November 2017; Accepted 11 December 2017; Published 2 January 2018

Academic Editor: Marc Thomas

Copyright (C) 2018 Gabriel Pino et al. This is an open access article distributed under the Creative Commons Attribution License, which permits unrestricted use, distribution, and reproduction in any medium, provided the original work is properly cited.

\begin{abstract}
The contribution of a medium-sized hydro power plant to the power grid can be either at base load or at peak load. When the latter is the most common operation mode, it increases the start and stop frequency, intensifying the hydro turbine components' degradation, such as the guide bearings. This happens due to more frequent operation in transient states, which means being outside the service point of the machines' nominal condition, consisting of speed, flow, and gross head. Such transient state operation increases the runner bearings' mechanical vibration. The readings are acquired during the runner start-ups and filtered by a DC component mean value and a wavelet packet transform. The filtered series are used to estimate the relationship between the maximum orbit curve displacement and the accumulated operating hours. The estimated equation associated with the ISO 7919-5 vibration standards establishes the sojourn times of the degradation states, sufficient to obtain the transition probability distribution. Thereafter, a triangular probability function is used to determine the observation probability distribution in each state. Both matrices are inputs required by a hidden Markov model aiming to simulate the equipment deterioration process, given a sequence of maximum orbit curve displacements.
\end{abstract}

\section{Introduction}

Hydro power plants (HPPs) are generally expected to run either at base load or at peak load. The former condition means they should operate uninterruptedly, irrespective of the interconnected grid's instantaneous demand variation, due to their lower marginal costs. Most electricity flowing in national interconnected grid (NIG) is generated by large HPPs. In addition, peak demand is usually supplied by thermal power plants (TPPs) and small HPPs, due to their higher marginal costs and lower energy efficiency. In theory, HPPs should only perform a normal shutdown during planned maintenance and for reservoir water level control. The continuous operation of large HPPs near the best efficiency point (BEP) demands low intermittency, which in turn depends on the reservoir gross head constancy, regular rainfall periods, and rigorous maintenance plans [1]. The national system operator (NSO) forces all Brazilian
HPPs to start, shut down, or change their generated power to attend the grid's instantaneous requirements. During a system demand variation, runners can assume different operation states: static, start-up, no-load, low-load, nominalload, and shut down. Start-up is one of the most damaging states regarding runner operating condition $[2,3]$.

Depending on the HPP's operation regime, its power unit components can be subject to greater degradation. A stressful start-stop cycle makes the runner operate in transient state many times, increasing degeneration of mechanical elements. Off-design conditions, such as unit start-stop, switching between operation regimes, load rejection, and out-of-phase synchronization, can influence rotors' transient operations $[4,5]$. The aging generated by electrical, thermal, and environmental stress due to excessive starts and stops can be compared with the years of operation and the estimated annual equivalent cost of reduced remaining lifetime [6]. There are four main failure modes related to volatile HPP 
conditions: cavitation, erosion, material defects, and fatigue [7]. For instance, pressure fluctuation due to rotor-stator interaction and occurrence of vortex rope in the draft tube at partial load operation are hydrodynamic effects addressed by researchers [8].

Orbit curve diagrams have been used in conditionbased maintenance (CBM) studies. They are included among the instruments used for vibration measurement and signal processing techniques for condition monitoring of machine tools in manufacturing operations $[9,10]$. For instance, orbit curves were used in the evaluation of the dynamic behavior of power units affected by steam-whirl instability, a common phenomenon in steam turbines caused by a rise of the steam flow and pressure $[11,12]$.

It is important to predict degradation of HPP's components before they reach failure limits. It is also valuable to know how the system operation and the degradation process are related. This paper focuses on one of the most important rotating machinery components, the turbine guide bearing [13]. During start-ups, they are submitted to hydraulic transient state [14]. This phenomenon is associated with mechanical vibrations that lead to a higher shaft surface displacement and a likely guide bearing trimming process. Continuous start-stop cycles cause progressive degradation of rotating elements. Shaft vibration measurement is essential to understand and quantify the entire process, so vibration monitoring systems are commonly installed in power units' bearings. These systems usually have limited resources regarding digital-to-analogue sampling frequency, memory buffer register and offset error induction. This lowers the quality measurements due to the presence of noise in a significant part of the information. More details are given in Section 3.2 and described in [15-18].

The acquired transient vibration waveforms are nonstationary and present low signal-to-noise ratio (SNR). These original noisy signals are unsuitable to analyze and design orbit curves, mainly for two reasons: high DC levels distort the orbit curve formation from the origin of the Cartesian plane; and low SNR signals do not properly represent the axis displacement due to vibration sparks and undesired harmonic content. Section 3.1 shows the difference of applying filtering techniques to better interpret the transient vibration behavior. The nonstationary waveforms require sophisticated filtering tools to handle time and frequency dependent data $[19,20]$. There are several mathematical methods to deal with vibration signals. They have chronologically evolved from frequency-domain analysis regarding digital filters [21] to wavelet transform denoising [22]. The wavelet transform method, applied in this paper, has the mathematical mechanisms to properly denoise and process the primary data acquired.

The filtered vibration database is accurate to develop a statistical method to predict and track the bearing degradation process. The signal processing applied to the database results in higher SNR and better represents the axis displacements as described in Section 4.1. The stochastic phenomenon evolves from "good as new" to "imminent failure." The state progression moves through adjacent states, in such way that the next state only depends on the current one, characterizing a Markov process. Markovian models are suitable for dividing equipment conditions into any meaningful state, facilitating general understanding of the specification of the process model [23]. Furthermore, they can characterize the stochastic relationship between the features extracted from condition monitoring data and the actual health states of the equipment [24].

Nevertheless, most of the time the bearing degradation state is not detectable because it is not possible to perform inspections during the HPP operation. So, the degradation process cannot be followed directly, meaning evolution is hidden. To overcome this limitation, vibration measurements are used as an observable variable indicating the degradation states of the bearing. The entire process can be statistically better represented as a hidden Markov model (HMM) [2528]. Besides the proposed purpose, HMM has other successful applications in speech recognition [29-31], monitoring of machine conditions, and fault diagnosis $[32,33]$.

This paper develops a procedure that does not require extensive historical vibration data to perform statistical assessment. The model also incorporates previous technical experiences acquired during preventive and corrective maintenance performed at the power plants and these parameters determine the decision model for maintenance based on the condition-based maintenance (CBM) of the equipment. It is possible to ascertain the guide bearing deterioration condition, for instance, based on available measurements and the proportional vibration limits established in ISO 7919$5[34,35]$. After the theoretical background presented in Section 2 and the technical development in Section 3 regarding vibration and stochastic methods, Section 4 discusses and applies the proposed method to measurements obtained from generating unit 1 (GU-1) of Corumbá IV HPP, located in Brazil, and finally Section 5 presents the conclusion.

\section{Theoretical Background}

The use of techniques for prognosis of bearing degradation should be divided into two parts: data acquisition and the data processing [36]. When considering data acquisition, this study performs a nonstationary signal analysis for bearing fault diagnosis. Feng et al. [37] classified the various time-frequency analysis methods applied to machinery fault diagnosis into four categories: linear and bilinear timefrequency representations (e.g., wavelet transform); adaptive parametric time-frequency analysis; adaptive nonparametric time-frequency analysis (e.g., Hilbert-Huang transform) [38]; and time varying higher order spectra. Various methods adapted to specific conditions have been used since then. The nuisance attribute projection (NAP) is introduced in the bearing performance degradation assessment to mitigate the influence of problems irrelevant to the degradation state introduced by operation conditions [25]. The Rényi entropy based features exploit the idea that a progressing fault implies rising dissimilarity in the distribution of energies across the vibrational spectral band sensitive to the bearing faults [39]. Envelope analysis has been applied to overcome the constraint of constant operating speed of the rolling element bearing. The squared envelope spectrum has been extended 

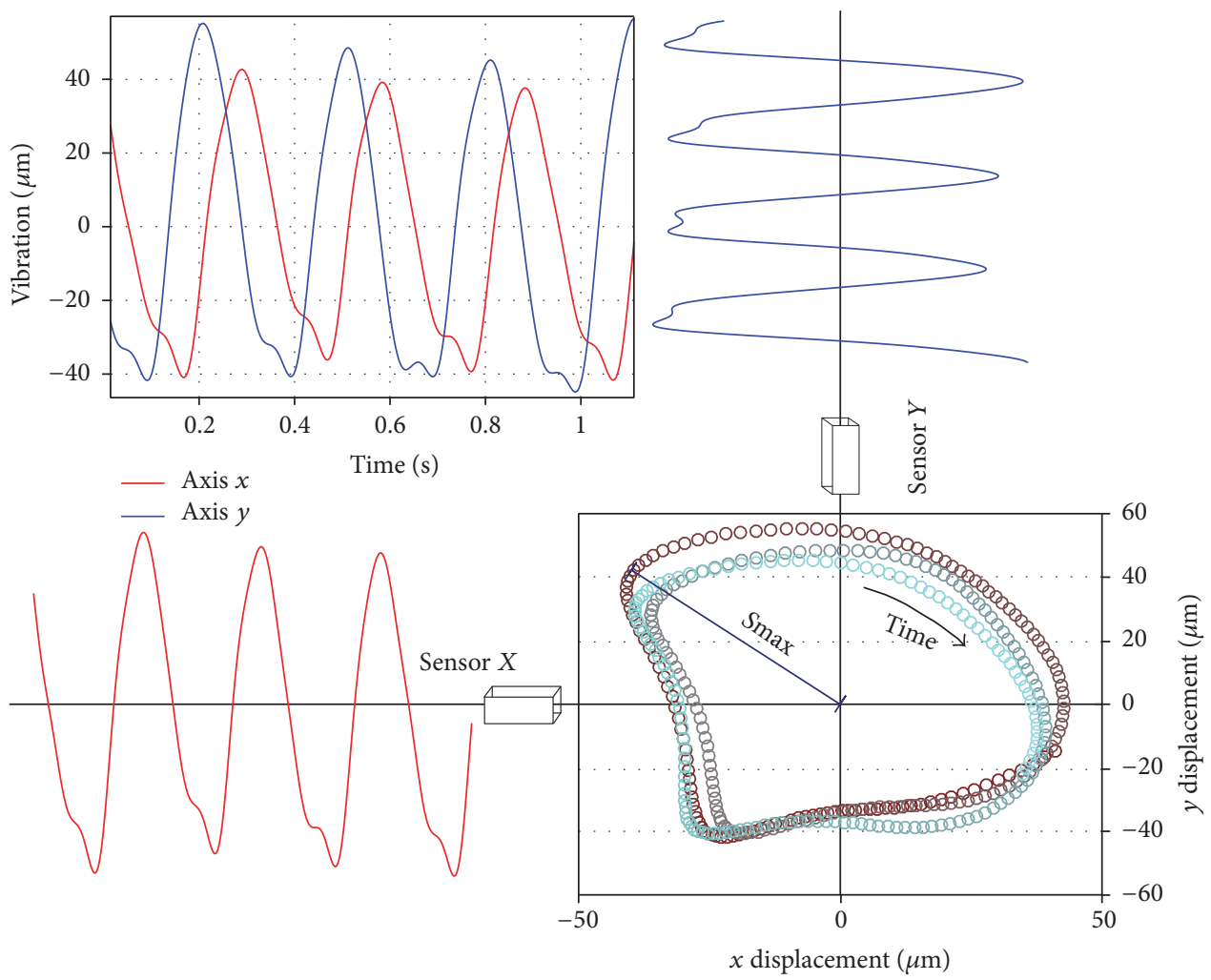

FIgURE 1: Sensors displacement and measurements.

to cases in which small speed fluctuations occur [40,41]. The spectral kurtosis technique adopts the concept of kurtosis to capture the impulsiveness of a signal. It uses a combination of short-time Fourier transform-based SK, kurtogram, adaptive SK, and protrugram [42]. However, despite such specific situations, the wavelet transform has been the most popular denoising technique for the extraction of the defect vibratory signature from the measured signal in which the random noise and other parameters of the bearing are immersed [4345]. With respect to data processing, the stochastic process inherent to bearing wear can be classified as a single component which depends on the nature of the degradation state: discrete or continuous [46]. When the technical condition of a bearing cannot be described by a continuous, measurable value, its deterioration is often assessed by visual inspections or by other methods that only lead to qualitative results. As an alternative to continuous measures, several discrete deterioration states can be defined [47]. Markov processes are used in such cases when measurement of discrete degradation states is not precise.

\section{Materials and Methods}

The reasons why a guide bearing degradation increases are strongly related to the shaft's axial displacement. This leads to possible relationship between the shaft vibration intensity and the deterioration caused by mechanical contact of rotating and static elements. Vibration measurements and degradation status are so closely related that many power plants have installed vibration monitoring systems to detect its evolution [48]. This type of system records a substantial increase of vibration level during hydro power units' startup, which takes approximately five minutes, depending on speed governor parameterization [49]. The vibration data acquired during these events are crucial to understand its consequences to the whole rotating group, including the evolution of the guide bearings' degradation.

3.1. Data Acquisition. The power unit shaft's mechanical vibration usually presents two signal sources, orthogonally installed, as shown in Figure 1. The configuration of both signals permits parameterizing them so as to describe the external profile of the shaft. The graph of the signal parameterization and composition that suppresses the time dependency is generally called a Lissajous curve [50], and specifically for shaft surface displacement it is called an orbit curve $[51,52]$. This curve is fundamental to infer how much the shaft perimeter is displaced from its center line. This particular measurement, called $S_{\max }$, is the maximum axis vector distance and its measurement is also used as an indicator for technical standards [34]. It is calculated as in

$$
S_{\max }=\sqrt{\max \left([x(t)]^{2}+[y(t)]^{2}\right)}, \quad 0 \leq t \leq \frac{1}{n_{\mathrm{rpm}}},
$$

where $n_{\mathrm{rpm}}$ is the rated turbine speed in revolutions per minute. This article considers $S_{\max }$ a collusion variable of the entire vibration phenomenon per machine revolution 

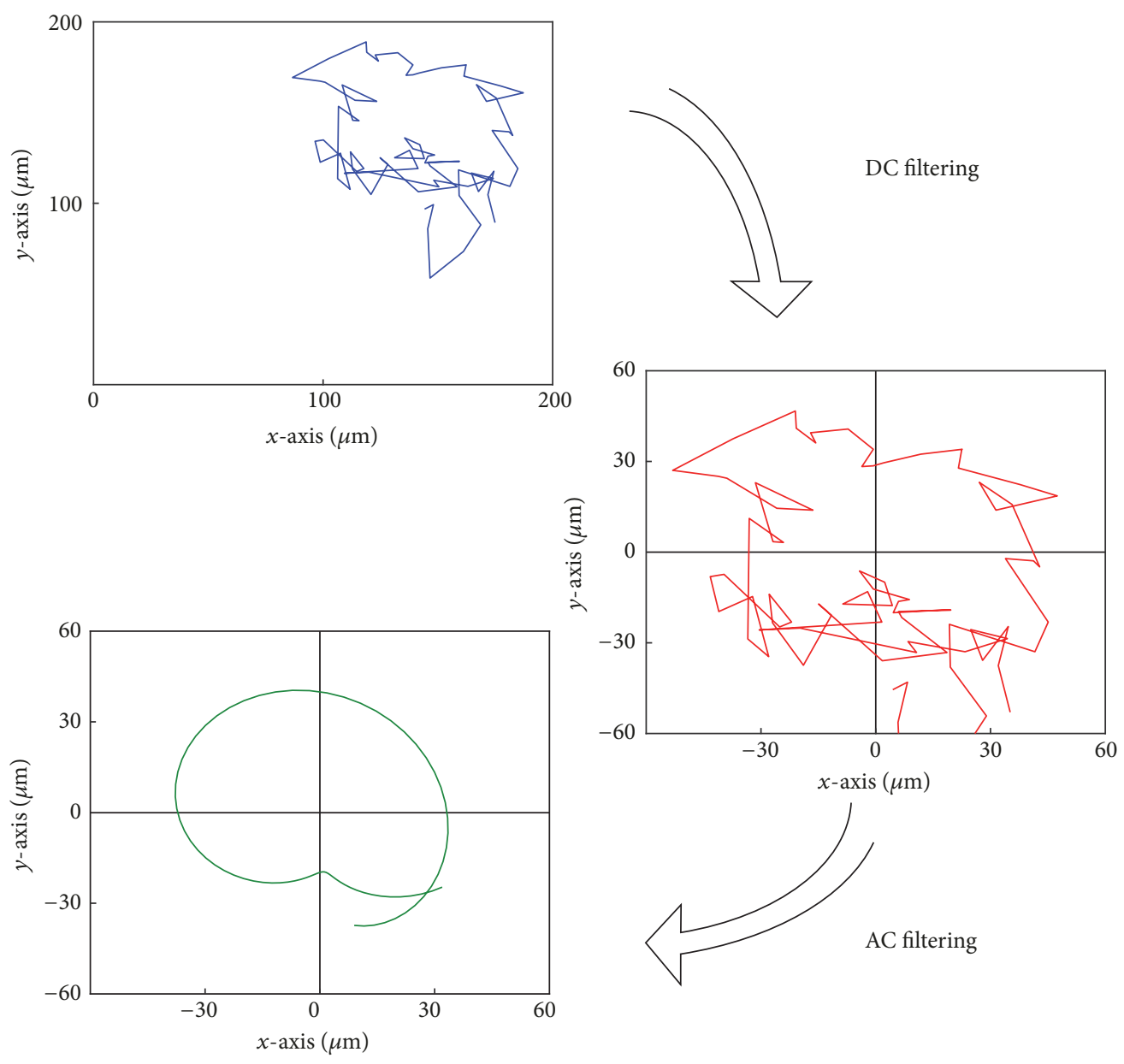

FIGURE 2: Orbit curve formation for different inputs quality.

in transient and steady states operation, so it is stored in a database for stochastic analysis.

Vibration monitoring systems installed in power plants usually use inductive proximity sensors to measure the distance between probe tips and the shaft surface [53]. Inductive sensors present low-pass filter response for displacement measurements; thus they physically attenuate high-frequency signals that could affect the vibration waveform analysis [54]. Besides this, most commercial electronic drives responsible for signal processing and analogue-to-digital converting operate with maximum frequency bandwidth up to $20 \mathrm{kHz}$ $[55,56]$. Another technical limitation of commercial systems is the memory buffers required to acquire long waveforms during five minutes and store the data. Lower frequency sampling implies worse information quality regarding vibration phenomenon due to the inherent presence of flicker noise [57]. Associated with this noise, HPP turbines usually present low rated speed, which implies their first harmonic is up to $10 \mathrm{~Hz}$, meaning that the fundamental signal frequency component is polluted by flicker and distributed thermal noise [58].

Technical issues related to data acquisition require signal processing using filtering techniques for better understanding of transient and steady state vibration waveforms.
3.2. Data Filtering. The technical limitations of vibration monitoring systems directly impact the precision and accuracy of orbit curve calculation. Hence, proper filtering tools must be applied to acquired waveforms. The signal processing depends on the correct mathematical approach given to each data characteristic, operation state, sensor accuracy, associated errors of analogue-to-digital converters, frequency sampling, SNR, noise distribution, and window lengths. Figure 2 illustrates the orbit curves formation regarding three different inputs: original vibration signals from the monitoring system, signals after DC filtering, and signals after DC and AC filtering. The first image does not properly represent the axis displacement and its center line is shifted from the origin. The second one is aligned to the center line but is still very noisy. The last one, after signal processing of the vibration inputs, better represents the axis displacement.

3.2.1. Frequency Spectrum. Frequency spectrum analysis is applied before choosing filtering tools in order to better understand the signal behavior and how its processing will be properly performed. This preliminary mathematical feature indicates the signal content and which harmonic components are the most important [59]. 
Specifically, the transient vibration signals acquired during machine start-ups present a nonstationary characteristic; that is, the frequency spectrum is not constant in the whole window analysis. This condition demands more general time and frequency-domain analysis such as short-time Fourier transform (STFT) [60]. Equation (2) shows the integral kernel dependency on time and frequency and (3) the Haning window function with period $\tau$.

$$
\begin{aligned}
\mathcal{S F}[x(t)] & =X(f, \tau)=\int_{-\infty}^{+\infty} x(t) e^{-j 2 \pi f t} W(t) d t, \\
W(t) & =\frac{1}{2}\left(1-\cos \left(2 \pi \frac{t}{\tau}\right)\right) .
\end{aligned}
$$

Using the time and frequency excursion of the STFT it is possible to infer a diverse frequency content of the AC component and also a DC level. The DC level content indicates the sensor setup before the data acquisition. The intrinsic differences between the original AC and DC vibration components require different mathematical tools to properly filter them.

3.2.2. DC Component Filtering. Orbit curve calculation necessarily uses peak-to-peak vibration values. Otherwise they would be shifted from the origin of the Cartesian plane and the waveform would be distorted, as can be inferred by (1) and seen in Figure 1. So the DC component must be filtered. The spectrum calculated by (2) possibly indicates a DC content during transient and steady states, which can be interpreted as the stand still distance between the shaft surface and the inductive sensor probe [55].

There are many techniques that can filter the DC component such as digital FIR or IIR high pass filtering [61]. However, these techniques present amplitude and phase frequency response. The behavior of a very low cutoff frequency filter implies nonlinear phase response near the inflection, which is not desirable since the fundamental frequency is usually up to $10 \mathrm{~Hz}$. In order to avoid phase shifting, this paper uses the mean vibration value as the DC reference.

3.2.3. AC Component Filtering. The vibration measurement during the runner start-up tends to assume nonstationary behavior, as can also be inferred using (2). This type of signal is not properly filtered by applying conventional techniques such as impulse response filtering. Wavelet transform is a very efficient denoising tool able to adapt the original waveform characteristics by means of frequency and amplitude modulation [62]. Equation (4) denotes the wavelet transform of a continuous signal $x(t)$. Although the acquired signals are discrete, the continuous expression better exemplifies the advantages of using the wavelet packet.

$$
\mathscr{W}[x(t)]=X(a, b)=\frac{1}{\sqrt{a}} \int_{-\infty}^{+\infty} x(t) \psi\left(\frac{t-b}{a}\right) d t
$$

where $\psi=\psi(t)$ is the mother wavelet function, $a$ is the frequency dilatation parameter and $b$ is the shifting parameter related to signal excursion in time-domain. The kernel composition of time and frequency parameters enables the integral transform to adapt them to the original signal characteristics, which is desirable for nonstationary measurements.

After the signal processing, the vibration data and the shaft replacement are ready to be statistically analyzed.

\subsection{Degradation Prediction Model}

3.3.1. Theoretical Limits for $S_{\max }$. According to ISO 7919-5, there is a relationship between $S_{\max }$ at the nominal machine operation and the guide bearing deterioration stage, depending on machine nominal speed. The vibration magnitudes can be related to four zones, which are defined as follows. The vibration of newly commissioned machines would normally fall within zone $\mathrm{A}$. In zone $\mathrm{B}$, machines are usually considered acceptable for unrestricted long-term operation. Machines with vibration within zone $C$ are ordinarily considered unsatisfactory for long-term continuous operation. Generally, the machine can be operated for a limited period in this condition until a suitable opportunity arises for maintenance. In zone D, vibration values are normally considered to be of sufficient severity to cause damage to the runner. These limits define the degradation states and the observable vibration limits in the Hidden Markov Model.

3.3.2. Triangular Probability Function. The association of a discrete nominal scale represented by linguistic expressions like "small deterioration" with a continuous degradation process is a fertile field for errors of judgment. Since $S_{\max }$ is a discrete observed variable associated with each state emission, it imbeds an imprecision related to both the nominal classification and the quality of the observed variable acquisition and filtering. A simple way to mitigate such imprecision is to work with intervals, in which case a symmetric triangular probability distribution is proposed. In this representation, the observed variable is defined by a set of three numbers $(L, M, U)$. These numbers are determined by $M$, a discrete value reflecting the calculated $S_{\max }$, and the interval, a sensitive value used to obtain the lower $(L)$ and upper $(U)$ bounds. The higher the degree, the higher the perceived imprecision regarding the observed variable. The triangular-density function is customarily used when function parameters are subjective. A typical example is for estimating activity durations by identifying a minimum, most likely, and maximum values, representing uncertain activity durations [63]. It has been shown that the cutoff points of such triangular function contain the confidence intervals of any symmetric probability distribution with the same mode and support [64].

3.3.3. Hidden Markov Model. Markov chains are stochastic processes in which the progression to the next state depends exclusively on the previous one [29]. The Hidden Markov Model (HMM) is a Markov progression generally observed indirectly, as a function of the transitions among states of the Markov chain governing process, and in which successive state transitions are determined by the likelihood model transition [29, 31-33, 65-68]. Since inspections are not 


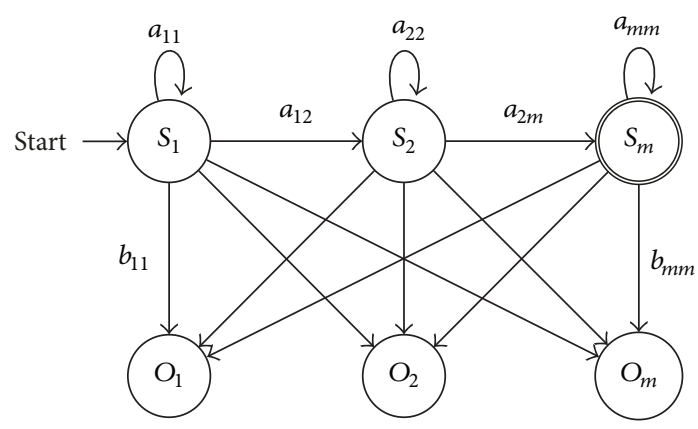

Figure 3: Emitted and observable states in HMM.

carried out continuously, unlike continuous and progressive equipment deterioration, the states cannot be observed, and as a result a Hidden Markov Model applies. Figure 3 demonstrates a hidden Markov process. In this case, one or more states elements $S_{1}, S_{2}, \ldots, S_{M}$ represent the degradation states and they are not directly observable. However, they emit observations $\mathrm{O}_{1}, \mathrm{O}_{2}, \ldots, \mathrm{O}_{M}$ that can be identified and associated with the states.

The elements $a_{i j}$ refer to the transition probabilities between states. For instance, $a_{11}$ is the probability that the process at time $T+1$ remains in state $S_{1}$ knowing that at time $T$ it was in state $S_{1}$. For machine degradation, the transitions between states do not allow the return to the previous state or the passing to the next state. This means the degradation of the equipment from one moment $T$ to the next $T+1$ will only stay in the home state or it will switch to the next. Thus, for three states, the transition matrix assumes

$$
T=\left[\begin{array}{ccc}
P_{11} & P_{12} & 0 \\
0 & P_{22} & P_{23} \\
0 & 0 & 1
\end{array}\right] .
$$

It can be observed that $P_{33}=1$; that is, once the last degradation state is reached, a potential failure condition occurs and corrective maintenance of the equipment is necessary. So the process is reset and the state is reestablished to "good as new." Based on the sojourn times, it is possible to estimate the transition matrix by the Bayesian method. The elements $b_{i j}$ show the emission probabilities. These are the probabilities with which the signals of the observable sequence are produced in each of the states. For example, state $S_{1}$ can emit several values for the observable signal: the probability of issuing $O_{1}$ is represented by $b_{11}$, of issuing $O_{2}$ by $b_{22}$ and so on. In addition, the model has as initial distribution of states $\pi_{i}$, where $\pi_{i}=P\left(S_{1}=i\right)$ for $1 \leq i \leq M$. The HMM process provides ways to perform the task of learning the parameters; that is, given a sequence of results, the algorithms estimate by maximum likelihood the transition and emission probabilities that best represent them. Figure 4 summarizes the proposed methodology.

\section{Results and Discussion}

The method presented in the previous section is applied as a case study to a power unit of Corumbá IV, which is
TABLE 1: Corumbá IV HPP main technical characteristics.

\begin{tabular}{lc}
\hline Number of power units & 2 \\
Rated turbine power & $64.8 \mathrm{MW}$ \\
Turbine diameter & $3287 \mathrm{~mm}$ \\
Rated turbine speed & $200 \mathrm{RPM}$ \\
Net head & $65 \mathrm{~m}$ \\
Reservoir capacity & 3.8 billion m \\
\hline
\end{tabular}

a medium-sized hydro power plant located in Brazil. Its main technical characteristics are reported in Table 1. The runner of Corumbá IV was chosen for the case study due to previous technical issues regarding the accelerated guide bearing degeneration process, which required corrective maintenance on August 16, 2015.

From August 2016 to May 2017, 14 measurements were acquired by the vibration monitoring system during the runner start-up. The method depicted in Figure 4 is applied to these acquisitions.

4.1. Data Processing. It is strongly recommended to acquire the start-up vibration measurement from stand still until the no-load speed condition, to assure complete acquisition of vibration data. The process takes approximately five minutes. According to the technical data sheet of the vibration monitoring system [55], the maximum sampling rate is $128 \mathrm{~Hz}$ regarding a five-minute memory buffer. Obeying the Nyquist criteria, the information is completely determined up to $64 \mathrm{~Hz}$, as can be inferred in the next subsection.

4.1.1. Frequency Spectrum. Applying (2), for a $2 \mathrm{~Hz}$ Haning window, the result is pictured in Figure 5. The surface relates the normalized vibration power density $V=V(f, t)$ to the excursion in time $t$ and frequency $f$, given in minutes and hertz, respectively. The vibration function domain is $D_{V}=$ $\left\{(f, t) \in \mathfrak{R}^{2} \mid 0 \leq f \leq 64,0 \leq t \leq 5\right\}$.

The normalized absolute value given by its image presents clear nonstationary behavior due to its nonconstant frequency arrangement. The hill over low frequencies presents decay up to $10 \mathrm{~Hz}$, suggesting an intense flicker noise content due to the signal's low sampling frequency [57]. Besides this, there are some random noise clouds over various frequencies of the AC component, even over the fundamental harmonic, which indicate distributed thermal noise pollution. There is also a constant DC level even after steady state is achieved, where the harmonic frequencies all act in the same direction, in approximately $250 \mathrm{~s}$.

4.1.2. DC Component Filtering. The DC filtering techniques discussed in Section 3.2.2 are compared using a FIR filter and the mean value. Table 2 shows the amplitude of the DC components filtered by each method and their relative difference. The comparison infers that their relative differences are not substantial, so the mean value technique is adopted as an advantage, by not distorting the phase frequency response.

4.1.3. AC Component Filtering. Figure 5 shows random noise over the entire surface domain of the unfiltered signal. The 
TABLE 2: DC filtering techniques result.

\begin{tabular}{lccc}
\hline Acquisition & Mean value & FIR filter & Difference \\
\hline $16 / 04 / 2017$ & $1089.76[\mu \mathrm{m}]$ & $1087.34[\mu \mathrm{m}]$ & $0.22[\%]$ \\
$02 / 05 / 2017$ & $1023.05[\mu \mathrm{m}]$ & $1022.54[\mu \mathrm{m}]$ & $0.05[\%]$ \\
$20 / 03 / 2017$ & $1018.53[\mu \mathrm{m}]$ & $1018.09[\mu \mathrm{m}]$ & $0.04[\%]$ \\
$23 / 05 / 2017$ & $1035.73[\mu \mathrm{m}]$ & $1036.67[\mu \mathrm{m}]$ & $0.09[\%]$ \\
$09 / 09 / 2016$ & $1063.90[\mu \mathrm{m}]$ & $1063.50[\mu \mathrm{m}]$ & $0.04[\%]$ \\
$17 / 04 / 2017$ & $1064.40[\mu \mathrm{m}]$ & $1063.52[\mu \mathrm{m}]$ & $0.08[\%]$ \\
\hline
\end{tabular}

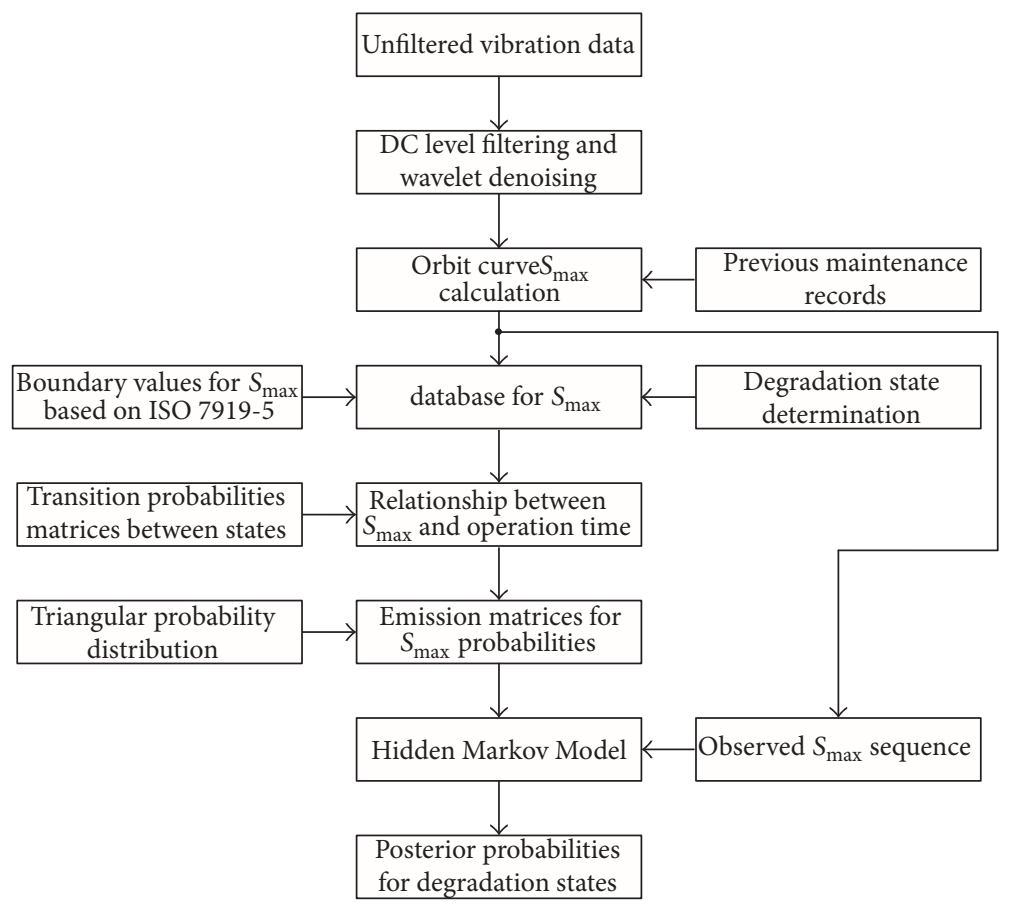

FIGURE 4: Methodology flowchart.

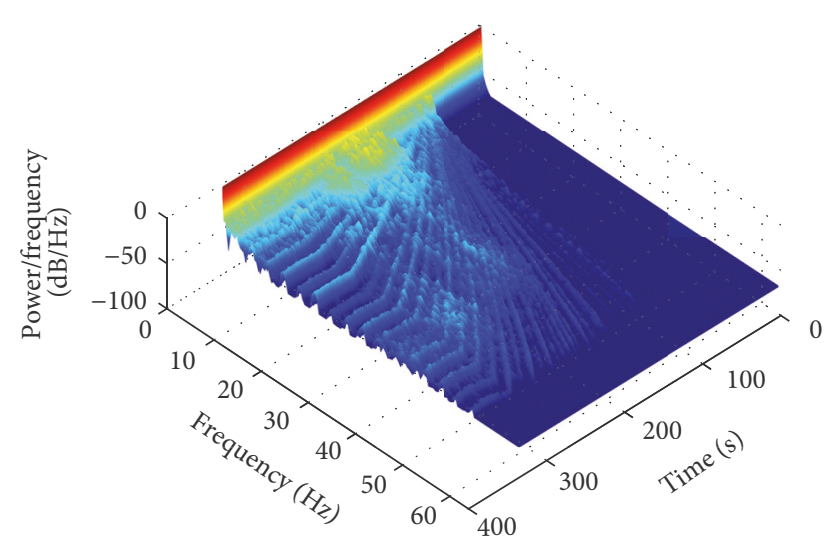

Figure 5: Short term Fourier transform of a transient state vibration.

prominent flicker noise is more concentrated in regions up to $10 \mathrm{~Hz}$ and the thermal noise power density is well distributed in the whole frequency excursion. The wavelet packet used for denoising presents the Symlet 8 mother wavelet due to its good performance, with quasisinusoidal waveforms, and the denoising process considers soft thresholding at seven frequency levels. Table 3 shows a SNR comparison before and after the signal processing for some frequency harmonics [69, $70]$. It can be noted that the signal processing considerably increased the relative signal power component over the noisy one. Figure 6 compares the original and filtered signals. Zooming reveals a large difference between each waveform, thus good adherence and filter behavior.

4.1.4. Sensitivity Analysis between $S_{\max }$ and Operation Time. The maximum displacement evolution over the operating time fits an exponential function nicely, as depicted in Figure 7. In a smoothing process, when the mean extracted from a subset containing the $k$ greatest numbers of a sample is its statistic, the exponential adherence among the statistics of the 14 samples is validated. Such confirmation has an important consequence on practical grounds, since the exponential relation is preserved even when two or more greater numbers are considered, mainly from the presence of outliers. This paper considers the relationship function for the maximum absolute value, $k=1$, over operation time, as observed in Table 4. 
TABLE 3: SNR comparison.

\begin{tabular}{lccc}
\hline Harmonic & Frequency $[\mathrm{Hz}]$ & SNR before & SNR after \\
\hline 0 & 0 & 37.39 & 317.62 \\
1 & 3.33 & 35.68 & 304.38 \\
3 & 10 & 25.99 & 205.45 \\
6 & 20 & 17.77 & 191.3 \\
8 & 26.66 & 15.16 & 132.9 \\
\hline
\end{tabular}

TABLE 4: $S_{\max }$ values over operation time.

\begin{tabular}{lccc}
\hline Acquisition & $k=1$ & $k=10$ & $k=20$ \\
\hline $09 / 09 / 2016$ & 149.92 & 147.71 & 146.59 \\
$19 / 12 / 2016$ & 166.45 & 163.65 & 158.91 \\
$06 / 01 / 2017$ & 169.22 & 161.93 & 156.87 \\
$19 / 01 / 2017$ & 171.97 & 170.36 & 166.24 \\
$20 / 02 / 2017$ & 178.09 & 175.69 & 170.64 \\
$21 / 02 / 2017$ & 176.28 & 174.07 & 170.95 \\
$20 / 03 / 2017$ & 183.35 & 180.22 & 174.52 \\
$05 / 04 / 2017$ & 186.39 & 183.62 & 179.97 \\
$16 / 04 / 2017$ & 188.74 & 181.79 & 177.00 \\
$17 / 04 / 2017$ & 185.51 & 182.72 & 177.15 \\
$24 / 04 / 2017$ & 190.84 & 183.91 & 178.70 \\
$02 / 05 / 2017$ & 191.62 & 183.55 & 176.31 \\
$23 / 05 / 2017$ & 196.98 & 191.45 & 184.93 \\
$30 / 05 / 2017$ & 197.68 & 189.71 & 180.82 \\
\hline
\end{tabular}

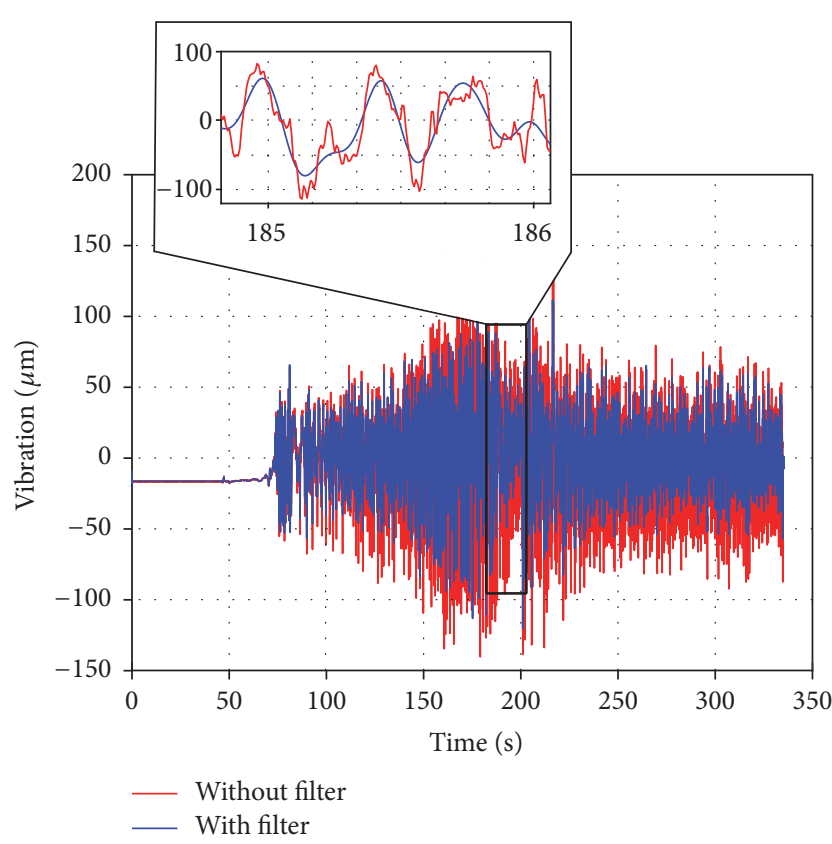

FIGURE 6: Comparison between original and filtered signals.

\subsection{Degradation Model}

4.2.1. Degradation Model Inputs. The runner nominal speed is $200 \mathrm{RPM}$, and according to [34], the $S_{\max }$ theoretical limits for a 200 RPM machine are shown in Table 5 . In this

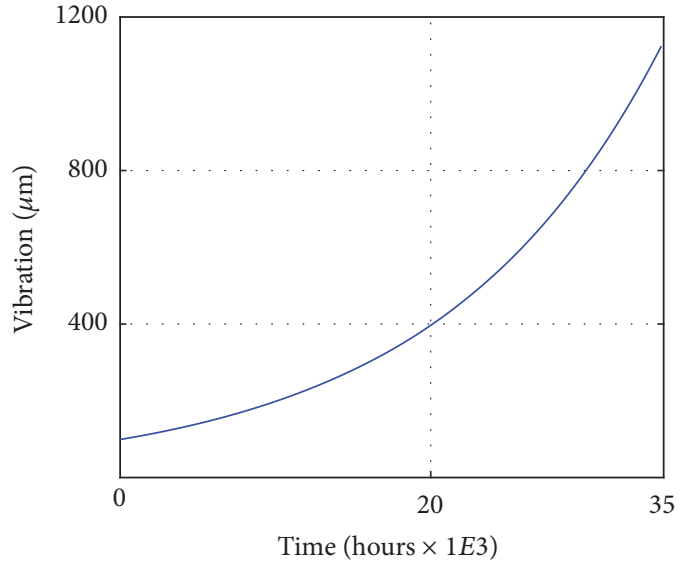

FIgURE 7: Exponential adherence between $S_{\max }$ and operation time.

case study, the $\mathrm{B}$ and $\mathrm{C}$ bands of minimum and maximum degradation, respectively, are split into 5 and 15 levels. This implies increments of $10.8 \mu \mathrm{m}$ for section B and $9 \mu \mathrm{m}$ for section $C$. The total number of states is divided into 22 subdivisions, 20 for $\mathrm{B}$ and $\mathrm{C}$ bands plus $\mathrm{A}$ and $\mathrm{D}$. This aims to refine the control bands, as well as improve the estimation of the proposed model probabilities. So, the degradation prediction model was developed based on readings of the vibration sensors. The cumulative hours of operation since the last preventive maintenance, which had reestablished good-as-new condition, were also recorded. The bearing 
TABLE 5: $S_{\max }$ limits for 200 RPM.

\begin{tabular}{lc}
\hline State & Maximum displacement $[\mu \mathrm{m}]$ \\
\hline A & $S_{\max }<82$ \\
B & $82 \leq S_{\max }<136$ \\
C & $136 \leq S_{\max }<271$ \\
D & $S_{\max } \geq 271$ \\
\hline
\end{tabular}

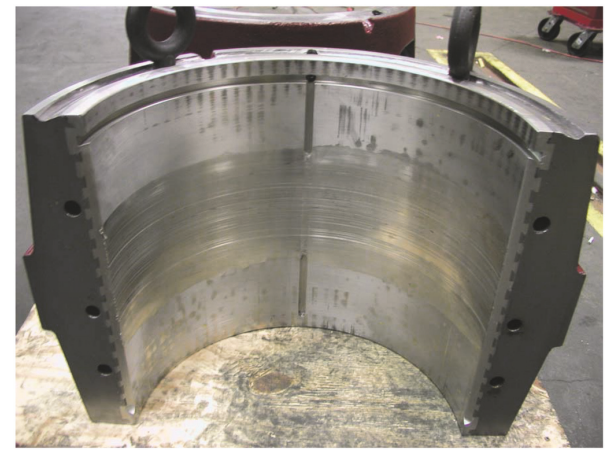

FIGURE 8: Guide bearing overview.

condition before the preventive maintenance is shown in Figure 8.

The deterioration process often accelerates as the operating time increases until reaching the remaining useful life. The expectation of the distribution of states in degradation prediction models presents features similar to those of exponential distributions [71]. The maximum displacement measurements of the Corumbá IV HPP by sensors follow this exponential behavior, which was used to calculate the sojourn times for the transition probabilities in the Markov Model.

4.2.2. Transition Probabilities. To simulate how the transitions states will evolve, an exponential curve is plotted, supported by the exponential behavior observed through the measurements. This exponential curve, shown in (6), relates the operating time to $S_{\max }$ and allows calculation of the sojourn times in each state according to the bands defined in the previous section.

$$
S_{\max }(t)=e^{3.1395924+0.00006944 t} \text {. }
$$

After calculating the sojourn times and knowing that on average Corumbá IV HPP operates 80.48 hours per start-stop cycle, it is possible to determine how many start-stop cycles, here called $n$, are associated with each state. This information is used to estimate the probability of remaining in the home condition. Since there is only one transition between each state, the probability of going to the next stage is $1 / n$ and of remaining in the home state is $1-1 / n$.

4.2.3. Emission Probabilities. In this case study, the estimated values for $S_{\max }$ are adopted as reference values for the observed variable. These values identify the change between states, and therefore must be linked to a probability distribution that binds them to the different states. Since the emissions are discrete and have a certain imprecision, due to the subjective nature of the degradation state, such boundaries are treated as probabilities. In this case, these sets are represented as a symmetric triangular probability function. Thus, for a given value of $S_{\max }$ treated as median, the lower and upper limits are determined according to this criterion. The link of ISO 7919-5 absolute limits with the corresponding linguistic terms of the degradation states may result in a certain level of vagueness, with the interval range having attenuation to such weakness. The decision about a fair value in $\mu \mathrm{m}$ for such range is based on sensitivity. When the value is too low, the emission probabilities are concentrated around few corresponding states and the ability to predict chances of being in some other near state is affected. When the value is too high, the emission probabilities are widely distributed around the most probable state causing insecurity to the decision maker. Therefore, different interval range values should be tested to define which value fairly reflects such level of imprecision.

4.2.4. Model Validation through Inspection and Maintenance Data. The plant's maintenance records showed that when the power unit operating time reached 24,000 hours, it underwent preventive maintenance. On this occasion, no anomaly was identified in the guide bearing and therefore this was judged subjectively by the technicians as if it still remained in operating condition. However, after operating 36,000 hours, the power unit underwent new preventive maintenance, when severe scratching of the coating metal was detected. As a result, the guide bearing was identified as being in a state of high deterioration and likely to compromise the regular operating condition of the power unit. The guide bearing was thus replaced and the equipment was classified as good as new again. This information about the guide bearing degradation identified in the 24,000 and 36,000 hour inspections was used to validate the model parameterization.

4.2.5. HMM Parameters Inference. Due to the characteristic of $S_{\max }$ progression, as shown in Figure 7, the left-right HMM $[29,72]$ is considered. This means that the degradation process is irreversible and once the equipment is in one specific state condition, it cannot move to the previous state anymore, remaining in the same state or advancing to the next one. The usage of HMM for diagnostics applies two complementary steps: estimation of the parameters and posterior probabilities. The former comprises assessment of both the transition and emission matrices. The $S_{\max }$ values obtained from the 14 vibration samples and the sojourn time of the states are used to estimate the transition probabilities. On the other hand, the emission probabilities feature the thresholds as stated by ISO 7919-5 and a symmetric triangular probability function. Both emission and transition probabilities are inputs of the inferred HMM model and are calculated by means of the forward-backward algorithm $[73,74]$. In the second phase, the HMM parameters are used to estimate the posterior probabilities given a sequence of two hypothetical $S_{\max }$ values. Following the forward and backward probabilities [29], each value represents the guide 
TABLE 6: Posteriori probabilities.

\begin{tabular}{|c|c|c|c|c|c|c|c|c|}
\hline \multirow{3}{*}{$S_{\max }[\mu \mathrm{m}]$} & \multicolumn{4}{|c|}{$T_{1}$} & \multicolumn{4}{|c|}{$T_{2}$} \\
\hline & \multicolumn{8}{|c|}{ State } \\
\hline & 19 & 20 & 21 & 22 & 19 & 20 & 21 & 22 \\
\hline $253-262$ & $24 \%$ & $76 \%$ & 0 & 0 & 0 & $93 \%$ & $7 \%$ & 0 \\
\hline $262-271$ & 0 & $27 \%$ & $73 \%$ & 0 & 0 & 0 & $93 \%$ & $7 \%$ \\
\hline$\geq 271$ & 0 & 0 & $79 \%$ & $21 \%$ & 0 & 0 & $71 \%$ & $29 \%$ \\
\hline
\end{tabular}

bearing degradation state at a specific time. In this case study, the observed $S_{\max }$ values are set hypothetically as 253,262 , and $271 \mu \mathrm{m}$, coincident with the boundaries of the 19th, 20th, and 21st degradation states, in a scale where the 1st state is "good as new" and the 22nd state is "imminent failure." The basic idea is to evaluate how responsive is the proposed model in estimating the degradation condition of the guide bearing, which is essential for the CBM.

Table 6 shows the posterior probabilities of being in states 19,20,21, or 22 at times of $T_{1}$ and $T_{2}$, relative to the vibration measurements onwards. These states are referred to as "excessive deterioration," "prefailure level 1," "prefailure level 2," and "imminent failure," respectively. The values for the operating times $T_{1}$ and $T_{2}$ depend on the state determined by $S_{\max }$, where the relationship is defined by (6). In the present simulation, the upper and lower limits of the triangular function sufficient to calculate the emission probabilities were set at $9 \mu \mathrm{m}$ relative to $S_{\max }$. In a first round, when two consecutive readings were 253 and $262 \mu \mathrm{m}$, the probability of having the degradation referred by state 20 after $T_{1}$ equal to 521.6 hours was $76 \%$ and after $T_{2}$ equal to 1,025 hours was $93 \%$. In a second round, since the next $S_{\max }$ reading increased to $271 \mu \mathrm{m}$, the probability of being in state 21 after $T_{1}$ equal to 503.4 hours was $73 \%$ and after $T_{2}$ equal to 989.8 hours was $93 \%$. In a third round, when the two consecutive readings were equal to $271 \mu \mathrm{m}$, the probabilities of having the degradation referred by states 21 and 22 after $T_{1}$ equal to 486.4 hours were $79 \%$ and $21 \%$, respectively. In this round, the probabilities of being in the same states 21 and 22 after $T_{2}$ equal to 972.8 hours changed to $71 \%$ and $29 \%$, respectively.

\section{Conclusion}

The signal processing methods applied in this article, such as mean DC level and wavelet packet, presented fair performance in handling nonstationary data. Flicker and thermal noise were attenuated and the obtained signals presented high SNR. The resulting vibration values and the maximum axis displacement were consistent for use as inputs for the proposed stochastic model. The proposed method has some important aspects. First, the vibration readings are acquired in a real operational environment, which means application of standard vibration monitoring systems. While on the one hand this method has the downside of reading imprecision, on the other it enables the operator himself to collect the signals without external assistance. The adoption of HMM has two aspects, both important features of the proposed method. First, it is related to the main aspect of a Markov technique that allows the operator to carry out the guide bearing diagnosis without the need of putting the runner on hold to perform inspections. The second is concerned with the possibility of modeling guide bearing diagnosis by means of corrective maintenance records, therefore based on past experience. In the case of Corumbá IV HPP, the filtering and HMM parameters are adapted to a routine diagnosis system. In this case, the plant operator simply acquires the axial vibration signals during the runner start-up and feeds the model with such raw data. The automated routine calculates the probabilities of the guide bearing being at certain stages of degradation. Therefore, the proposed method has substantial advantages by reducing failure risk and maintenance costs.

\section{Conflicts of Interest}

The authors declare that there are no conflicts of interest regarding the publication of this paper.

\section{Acknowledgments}

The authors would like to acknowledge Corumbá Concessões S/A and ANEEL for their financial and operational support to this R\&D project.

\section{References}

[1] U. Seidel, C. Mende, B. Hübner, W. Weber, and A. Otto, "Dynamic loads in Francis runners and their impact on fatigue life," in Proceedings of the 27th IAHR Symposium on Hydraulic Machinery and Systems, IAHR 2014, Canada, September 2014.

[2] X. Liu, Y. Luo, and Z. Wang, "A review on fatigue damage mechanism in hydro turbines," Renewable \& Sustainable Energy Reviews, vol. 54, pp. 1-14, 2016.

[3] A. Coutu, M. Gagnon, and C. Monette, Life Assessment of Francis Runners Using Strain Gage Site Measurements, Waterpower $X V$, TN, Chattanooga, 2007.

[4] V. Iliev, P. Popovski, and Z. Markov, “Transient phenomena analysis in hydroelectric power plants at off-design operating conditions," International Journal of Engineering Research and Applications (IJERA), vol. 2, 2012.

[5] X. Huang, J. Chamberland-Lauzon, C. Oram, A. Klopfer, and N. Ruchonnet, "Fatigue analyses of the prototype Francis runners based on site measurements and simulations," in Proceedings of the 27th IAHR Symposium on Hydraulic Machinery and Systems, IAHR 2014, Canada, September 2014.

[6] T. P. Hara, L. F. L. Vianna, E. E. Nachvalger, and E. Goncalves, "Valuation about remaining service life of GBM hydraulic power plant units technical and economical aspects," in Proceedings of the IEEE 4th International Conference on Properties and Applications of Dielectric Materials, pp. 804-806, July 1994. 
[7] U. Dorji and R. Ghomashchi, "Hydro turbine failure mechanisms: An overview," Engineering Failure Analysis, vol. 44, pp. 136-147, 2014.

[8] K. C. Anup, B. Thapa, and Y.-H. Lee, "Transient numerical analysis of rotor-stator interaction in a Francis turbine," Journal of Renewable Energy, vol. 65, pp. 227-235, 2014.

[9] D. Goyal and B. S. Pabla, "Condition based maintenance of machine tools-a review," CIRP Journal of Manufacturing Science and Technology, vol. 10, pp. 24-35, 2015.

[10] D. Goyal and B. S. Pabla, "The vibration monitoring methods and signal processing techniques for structural health monitoring: a review," Archives of Computational Methods in Engineerin: State-of-the-Art Reviews, vol. 23, no. 4, pp. 585-594, 2016.

[11] P. Pennacchi and A. Vania, "Model based analysis of steam-whip instability onsets occurred in a power plant," in Proceedings of the 2009 ASME International Design Engineering Technical Conferences and Computers and Information in Engineering Conference, DETC2009, pp. 1025-1032, USA, September 2009.

[12] P. Pennacchi and A. Vania, "Analysis of the instability phenomena caused by steam in high-pressure turbines," Shock and Vibration, vol. 18, no. 4, pp. 593-612, 2011.

[13] S. Dong, S. Yin, B. Tang, L. Chen, and T. Luo, "Bearing degradation process prediction based on the support vector machine and Markov model," Shock and Vibration, vol. 2014, Article ID 717465, 15 pages, 2014.

[14] C. Trivedi, B. Gandhi, and C. J. Michel, "Effect of transients on Francis turbine runner life: A review," Journal of Hydraulic Research, vol. 51, no. 2, pp. 121-132, 2013.

[15] E. J. Siragusa and I. Galton, "Gain error correction technique for pipelined analogue-to-digital converters," IEEE Electronics Letters, vol. 36, no. 7, pp. 617-618, 2000.

[16] G. Conforti, M. Brenci, A. Mencaglia, and A. G. Mignani, "Fiber optic vibration sensor for remote monitoring in high power electric machines," Applied Optics, vol. 28, no. 23, pp. 5158-5161, 1989.

[17] G. Fang, "Whittaker-Kotelnikov-Shannon sampling theorem and aliasing error," Journal of Approximation Theory, vol. 85, no. 2, pp. 115-131, 1996.

[18] P. L. Butzer, G. Schmeisser, and R. L. Stens, "An Introduction to Sampling Analysis," in Nonuniform Sampling, pp. 17-121, Springer, Berlin, Germany, 2001.

[19] Z. Peng, F. Chu, and Y. He, "Vibration signal analysis and feature extraction based on reassigned wavelet scalogram," Journal of Sound and Vibration, vol. 253, no. 5, pp. 1087-1100, 2003.

[20] W. J. Wang and P. D. McFadden, "Application of wavelets to gear box vibration signals for fault detection," Journal of Sound and Vibration, vol. 192, no. 5, pp. 927-939, 1996.

[21] B. R. Murphy and I. Watanabe, "Digital shaping filters for reducing machine vibration," IEEE Transactions on Robotics and Automation, vol. 8, no. 2, pp. 285-289, 1992.

[22] Y. Wang, Z. He, and Y. Zi, "Enhancement of signal denoising and multiple fault signatures detecting in rotating machinery using dual-tree complex wavelet transform," Mechanical Systems and Signal Processing, vol. 24, no. 1, pp. 119-137, 2010.

[23] X.-S. Si, W. Wang, C.-H. Hu, and D.-H. Zhou, "Remaining useful life estimation-a review on the statistical data driven approaches," European Journal of Operational Research, vol. 213, no. 1, pp. 1-14, 2011.

[24] T. T. Le, F. Chatelain, and C. Berenguer, "Hidden markov models for diagnostics and prognostics of systems under multiple deterioration modes," in Proceedings of the in European Safety and Reliability Conference-ESREL, 2014, pp. 1197-1204, 2014.
[25] H. Jiang, J. Chen, and G. Dong, "Hidden Markov model and nuisance attribute projection based bearing performance degradation assessment," Mechanical Systems and Signal Processing, vol. 72-73, pp. 184-205, 2016.

[26] J. Yu, "Health condition monitoring of machines based on hidden markov model and contribution analysis," IEEE Transactions on Instrumentation and Measurement, vol. 61, no. 8, pp. 2200-2211, 2012.

[27] M. Yuwono, Y. Qin, J. Zhou, Y. Guo, B. G. Celler, and S. W. Su, "Automatic bearing fault diagnosis using particle swarm clustering and Hidden Markov Model," Engineering Applications of Artificial Intelligence, 2015.

[28] T. Boutros and M. Liang, "Detection and diagnosis of bearing and cutting tool faults using hidden Markov models," Mechanical Systems and Signal Processing, vol. 25, no. 6, pp. 2102-2124, 2011.

[29] L. R. Rabiner, "Tutorial on hidden Markov models and selected applications in speech recognition," Proceedings of the IEEE, vol. 77, no. 2, pp. 257-286, 1989.

[30] F. Jelinek, "Continuous Speech Recognition by Statistical Methods," Proceedings of the IEEE, vol. 64, no. 4, pp. 532-556, 1976.

[31] L. R. Rabiner and B.-H. Juang, "An introduction to hidden Markov models," IEEE ASSP Magazine, vol. 3, no. 1, pp. 4-16, 1986.

[32] Y. Xu, A. Xu, and T. Xie, "Two General Architectures for Intelligent Machine Performance Degradation Assessment," Shock and Vibration, vol. 2015, Article ID 676959, 2015.

[33] G. F. Wang, X. L. Feng, and C. Liu, "Bearing fault classification based on conditional random field," Shock and Vibration, vol. 20, no. 4, pp. 591-600, 2013.

[34] ISO, "Mechanical vibration of non-reciprocating machines $\Gamma$," measurements on rotating shafts and evaluation criteria, vol. 5, no. ISO 7919-5, 1997.

[35] J. M. Robichaud and P. Eng, "Reference standards for vibration monitoring and analysis," Bretech Engineering Ltd, vol. 70, 2009.

[36] A. K. S. Jardine, D. Lin, and D. Banjevic, "A review on machinery diagnostics and prognostics implementing condition-based maintenance," Mechanical Systems and Signal Processing, vol. 20, no. 7, pp. 1483-1510, 2006.

[37] Z. Feng, M. Liang, and F. Chu, "Recent advances in timefrequency analysis methods for machinery fault diagnosis: a review with application examples," Mechanical Systems and Signal Processing, vol. 38, no. 1, pp. 165-205, 2013.

[38] Z. H. Wu and N. E. Huang, "Ensemble empirical mode decomposition: a noise-assisted data analysis method," Advances in Adaptive Data Analysis (AADA), vol. 1, no. 1, pp. 1-41, 2009.

[39] P. Boškoski, M. Gašperin, D. Petelin, and Đ. Juričić, “Bearing fault prognostics using Rényi entropy based features and Gaussian process models," Mechanical Systems and Signal Processing, vol. 52-53, pp. 327-337, 2015.

[40] D. Abboud, J. Antoni, S. Sieg-Zieba, and M. Eltabach, "Envelope analysis of rotating machine vibrations in variable speed conditions: A comprehensive treatment," Mechanical Systems and Signal Processing, vol. 84, pp. 200-226, 2017.

[41] P. Borghesani, R. Ricci, S. Chatterton, and P. Pennacchi, "A new procedure for using envelope analysis for rolling element bearing diagnostics in variable operating conditions," Mechanical Systems and Signal Processing, vol. 38, no. 1, pp. 23-35, 2013.

[42] Y. X. Wang, J. W. Xiang, R. Markert, and M. Liang, "Spectral kurtosis for fault detection, diagnosis and prognostics of rotating machines: a review with applications," Mechanical Systems and Signal Processing, vol. 66-67, pp. 679-698, 2016. 
[43] C. Mishra, A. K. Samantaray, and G. Chakraborty, "Rolling element bearing defect diagnosis under variable speed operation through angle synchronous averaging of wavelet de-noised estimate," Mechanical Systems and Signal Processing, vol. 72-73, pp. 206-222, 2016.

[44] A. Tabrizi, L. Garibaldi, A. Fasana, and S. Marchesiello, "Early damage detection of roller bearings using wavelet packet decomposition, ensemble empirical mode decomposition and support vector machine," Meccanica, vol. 50, no. 3, pp. 865-874, 2015.

[45] Z. Zhang, Y. Wang, and K. Wang, "Fault diagnosis and prognosis using wavelet packet decomposition, Fourier transform and artificial neural network," Journal of Intelligent Manufacturing, vol. 24, no. 6, pp. 1213-1227, 2013.

[46] A. Alaswad and Y. Xiang, "A review on condition-based maintenance optimization models for stochastically deteriorating system," Reliability Engineering \& System Safety, vol. 157, pp. 5463, 2017.

[47] T. Welte, Deterioration and maintenance models for components in hydropower plants, Ph.D. dissertation [Ph.D. thesis], 2008.

[48] B. Rao, Handbook of Condition Monitoring, Elsevier, 1996.

[49] N. Kishor, R. P. Saini, and S. P. Singh, "A review on hydropower plant models and control," Renewable \& Sustainable Energy Reviews, vol. 11, no. 5, pp. 776-796, 2007.

[50] H. A. AL-Khazali, "Geometrical and Graphical Representations Analysis of Lissajous Figures in Rotor Dynamic System," IOSR Journal Of Engineering, vol. 02, no. 05, pp. 971-978, 2012.

[51] A. Muszynska, "Vibrational Diagnostics of Rotating Machinery Malfunctions," International Journal of Rotating Machinery, vol. 1, no. 3-4, pp. 237-266, 1995.

[52] N. Bachschmid, P. Pennacchi, and A. Vania, "Diagnostic significance of orbit shape analysis and its application to improve machine fault detection," Journal of the Brazilian Society of Mechanical Sciences and Engineering, vol. 26, no. 2, pp. 200-208, 2004.

[53] C. L. Prates and M. R. Siniscalchi, "Supervisory system for the turbine generator set of angra 1 npp," in Proceedings of the International Nuclear Atlantic Conference (INAC, '09), p. 7, 2009.

[54] C. J. Moore, "Frequency response corrections for eddy correlation systems," Boundary Layer Meteorology, vol. 37, no. 1-2, pp. 17-35, 1986.

[55] SKF, Eddy probe systems catalog, 2011.

[56] LionPrecision, Eddy current sensors for position and displacement with high speed and high resolution, 2016.

[57] J. A. Barnes and D. W. Allan, "A Statistical Model of Flicker Noise," Proceedings of the IEEE, vol. 54, no. 2, pp. 176-178, 1966.

[58] T. B. Gabrielson, "Mechanical-thermal noise in micromachined acoustic and vibration sensors," IEEE Transactions on Electron Devices, vol. 40, no. 5, pp. 903-908, 1993.

[59] N. Tandon and A. Choudhury, "Review of vibration and acoustic measurement methods for the detection of defects in rolling element bearings," Tribology International, vol. 32, no. 8, pp. 469-480, 1999.

[60] C. Kar and A. R. Mohanty, "Vibration and current transient monitoring for gearbox fault detection using multiresolution Fourier transform," Journal of Sound and Vibration, vol. 311, no. 1-2, pp. 109-132, 2008.

[61] N. Singer, W. Singhose, and W. Seering, "Comparison of filtering methods for reducing residual vibration," European Journal of Control, vol. 5, no. 2-4, pp. 208-218, 1999.
[62] Z. Yan, A. Miyamoto, and Z. Jiang, "Frequency slice wavelet transform for transient vibration response analysis," Mechanical Systems and Signal Processing, vol. 23, no. 5, pp. 1474-1489, 2009.

[63] W. E. Back, W. W. Boles, and G. T. Fry, "Defining triangular probability distributions from historical cost data," Journal of Construction Engineering and Management, vol. 126, no. 1, pp. 29-37, 2000.

[64] D. Dubois, L. Foulloy, G. Mauris, and H. Prade, "Probabilitypossibility transformations, triangular fuzzy sets, and probabilistic inequalities," Reliable Computing, vol. 10, no. 4, pp. 273297, 2004.

[65] F. Jelinek, Statistical Methods for Speech Recognition, MIT press, UK, 1997.

[66] B.-H. Juang, "On the hidden Markov model and dynamic time warping for speech recognition-a unified view," Bell Laboratories Technical Journal, vol. 63, no. 7, pp. 1213-1243, 1984.

[67] S. E. Levinson, "Structural Methods in Automatic Speech Recognition," Proceedings of the IEEE, vol. 73, no. 11, pp. 16251650, 1985.

[68] J. Makhoul, S. Roucos, and H. Gish, "Vector quantization in speech coding," Proceedings of the IEEE, vol. 73, no. 11, pp. 1551$1588,1985$.

[69] F. J. Harris, "On the use of windows for harmonic analysis with the discrete Fourier transform," Proceedings of the IEEE, vol. 66, no. 1, pp. 51-83, 1978.

[70] Y. Hu and P. C. Loizou, "Evaluation of objective quality measures for speech enhancement," IEEE Transactions on Audio, Speech and Language Processing, vol. 16, no. 1, pp. 229-238, 2008.

[71] T. M. Welte, J. Vatn, and J. Heggset, "Markov state model for optimization of maintenance and renewal of hydro power components," in Proceedings of the 2006 9th International Conference on Probabilistic Methods Applied to Power Systems, PMAPS, Sweden, June 2006.

[72] S.-Z. Yu, "Hidden semi-Markov models," Artificial Intelligence, vol. 174, no. 2, pp. 215-243, 2010.

[73] L. Himmelmann, Hmm-Hidden Markov Models, vol. 1 of $R$ Package Version, 2010.

[74] Y. Ephraim and N. Merhav, "Hidden Markov processes," Institute of Electrical and Electronics Engineers Transactions on Information Theory, vol. 48, no. 6, pp. 1518-1569, 2002. 


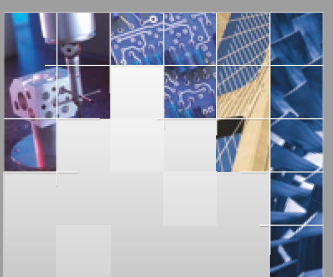

\section{Enfincering}
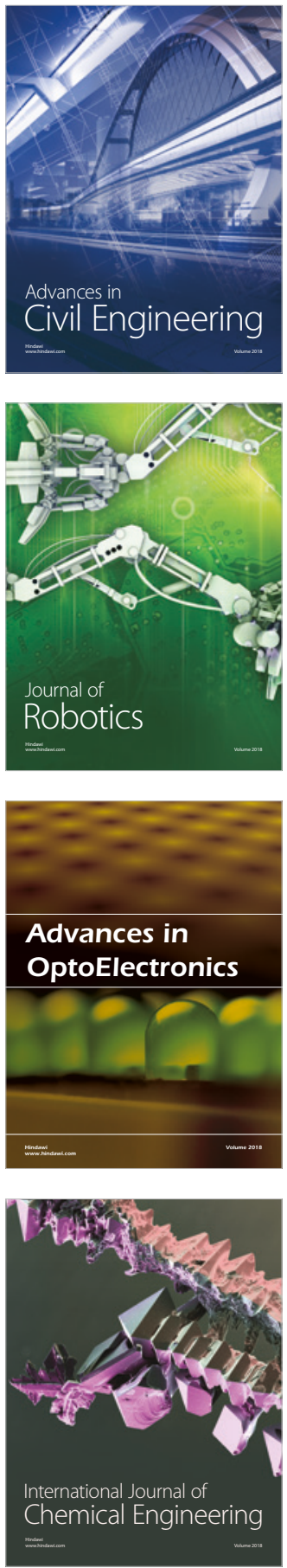

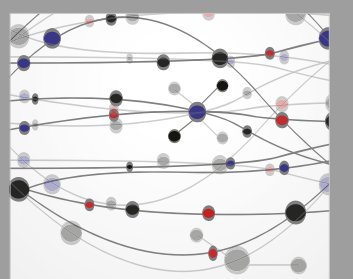

\section{Rotating \\ Machinery}

The Scientific World Journal

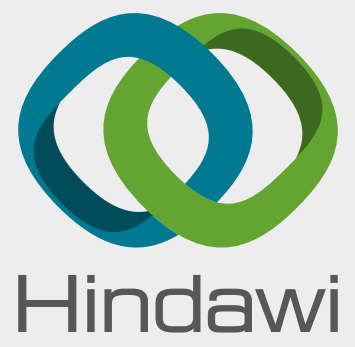

Submit your manuscripts at

www.hindawi.com
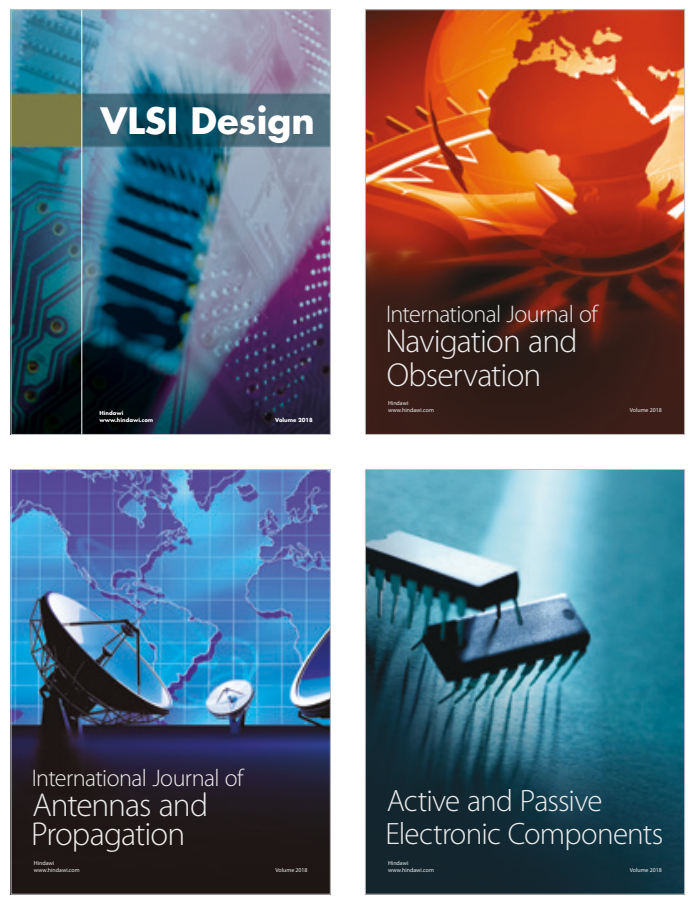
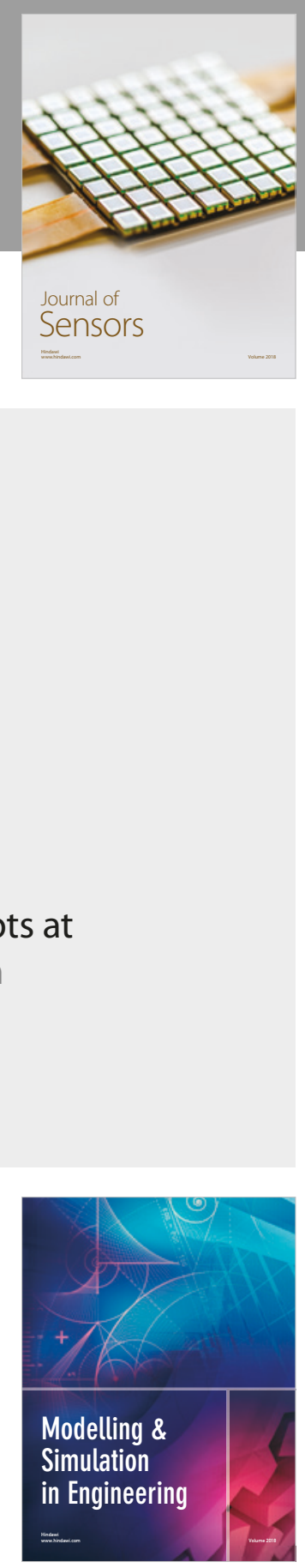

\section{Advances \\ Multimedia}
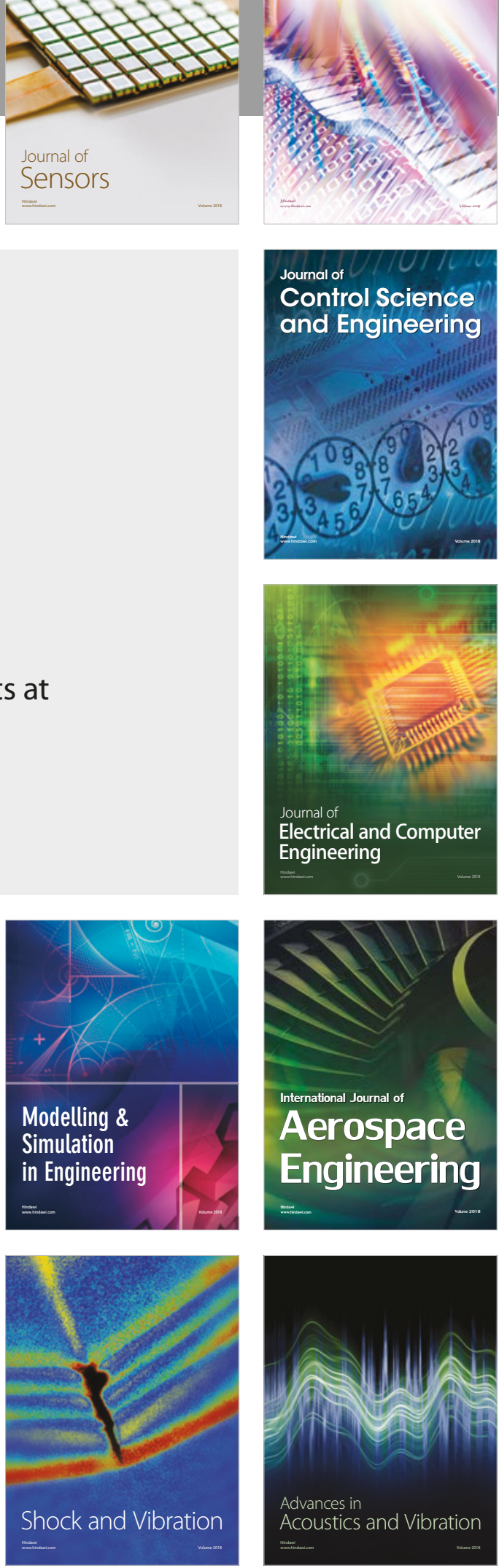